\title{
Carbon monoxide-Releasing Molecule-2 (CORM-2) attenuates acute hepatic ischemia reperfusion injury in rats
}

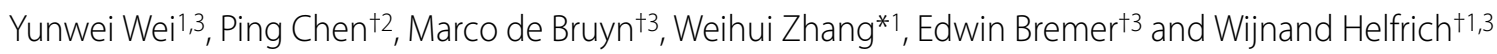

\begin{abstract}
Background: Hepatic ischemia-reperfusion injury (I/Ri) is a serious complication occurring during liver surgery that may lead to liver failure. Hepatic I/Ri induces formation of reactive oxygen species, hepatocyte apoptosis, and release of pro-inflammatory cytokines, which together causes liver damage and organ dysfunction. A potential strategy to alleviate hepatic I/Ri is to exploit the potent anti-inflammatory and cytoprotective effects of carbon monoxide (CO) by application of so-called CO-releasing molecules (CORMs). Here, we assessed whether CO released from CORM-2 protects against hepatic I/Ri in a rat model.

Methods: Forty male Wistar rats were randomly assigned into four groups $(n=10)$. Sham group underwent a sham operation and received saline. I/R group underwent hepatic I/R procedure by partial clamping of portal structures to the left and median lobes with a microvascular clip for 60 minutes, yielding 70\% hepatic ischemia and subsequently received saline. CORM-2 group underwent the same procedure and received $8 \mathrm{mg} / \mathrm{kg}$ of CORM-2 at time of reperfusion. iCORM-2 group underwent the same procedure and received iCORM-2 $(8 \mathrm{mg} / \mathrm{kg})$, which does not release CO. Therapeutic effects of CORM-2 on hepatic l/Ri was assessed by measuring serum damage markers AST and ALT, liver histology score, TUNEL-scoring of apoptotic cells, NFkB-activity in nuclear liver extracts, serum levels of proinflammatory cytokines TNF- $a$ and IL-6, and hepatic neutrophil infiltration.

Results: A single systemic infusion with CORM-2 protected the liver from I/Ri as evidenced by a reduction in serum AST/ALT levels and an improved liver histology score. Treatment with CORM-2 also up-regulated expression of the antiapoptotic protein $\mathrm{BCl}-2$, down-regulated caspase-3 activation, and significantly reduced the levels of apoptosis after I/ Ri. Furthermore, treatment with CORM-2 significantly inhibited the activity of the pro-inflammatory transcription factor NF-KB as measured in nuclear extracts of liver homogenates. Moreover, CORM-2 treatment resulted in reduced serum levels of pro-inflammatory cytokines TNF- $a$ and IL- 6 and down-regulation of the adhesion molecule ICAM-1 in the endothelial cells of liver. In line with these findings, CORM-2 treatment reduced the accumulation of neutrophils in the liver upon I/Ri. Similar treatment with an inactive variant of CORM-2 (iCORM-2) did not have any beneficial effect on the extent of liver I/Ri.
\end{abstract}

Conclusions: CORM-2 treatment at the time of reperfusion had several distinct beneficial effects on severity of hepatic I/Ri that may be of therapeutic value for the prevention of tissue damage as a result of I/Ri during hepatic surgery.

\section{Background}

Hepatic ischemia-reperfusion injury ( $\mathrm{I} / \mathrm{Ri})$ is a serious and common adverse event during hepatic surgery that may ultimately lead to liver failure, systemic inflamma-

\footnotetext{
* Correspondence: y.wei@chir.umcg.nl

1 Third department of General Surgery, First Clinical Hospital Harbin, Harbin

Medical University, Harbin 150001, Heilongjiang, China

+ Contributed equally

Full list of author information is available at the end of the article
}

tory response syndrome (SIRS) and even multiple organ failure syndrome (MOF) [1-4]. Central to hepatic $I / R i$ is the generation of reaction oxygen species (ROS) by activated Kupffer cells or neutrophils upon the reintroduction of molecular oxygen to ischemic tissues. This pathogenic event triggers a series of deleterious effects that include oxidative modification of lipids and proteins, induction of apoptosis in hepatocytes, release of pro- 
inflammatory cytokines, increased expression of adhesion molecules, and infiltration of leukocytes, which together leads to massive tissue destruction $[5,6]$.

To ameliorate the severity of liver $\mathrm{I} / \mathrm{Ri}$, several therapeutic strategies are currently being pursued, including the inhibition of apoptosis by decreasing cellular metabolism using the gas hydrogen sulphide $\left(\mathrm{H}_{2} \mathrm{~S}\right)$. Application of $\mathrm{H}_{2} \mathrm{~S}$ has shown promising activity in various pre-clinical I/Ri and transplantation models, including kidney and liver $[7,8]$. A second interesting strategy is to inhibit mitochondrial calcium overload, e.g. with 2-ABP [9], and thus block the execution of mitochondrial apoptotic signaling [10]. A third particularly appealing strategy is the use of anti-oxidants that directly counteract the deleterious effects of ROS. In this respect, dietary anti-oxidative supplements such as rutin and L-arginine have shown beneficial effects on severity of hepatic I/Ri [11]. Moreover, carbon monoxide $(\mathrm{CO})$ has raised particular therapeutic interest because of its potent anti-oxidant and antiinflammatory activity.

$\mathrm{CO}$ is best known as an odorless and toxic gas which upon inhalation binds with high affinity to heme, thereby forming carboxyhemoglobin and severely impairing the respiratory system. However, $\mathrm{CO}$ is also produced by the protein heme oxygenase $(\mathrm{HO})$ and as such functions as a potent endogenous antioxidant that counteracts toxic effects of ROS. HO-1 degrades heme into biliverdin, free iron, and $\mathrm{CO}$ [12] and is one of the most prominent lines of cellular defense against damage induced by $\mathrm{I} / \mathrm{Ri}$. Indeed, during oxidative stress the expression of the inducible form of $\mathrm{HO},(\mathrm{HO}-1)$ is strongly up-regulated in the liver [13].

Therapeutic up-regulation of $\mathrm{CO}$ tissue levels can be achieved via exogenous application of $\mathrm{CO}$, for instance by direct inhalation of CO gas. In ex vivo isolated liver perfusion and liver transplantation models exogenously added $\mathrm{CO}$ yields potent cytoprotective effects [14-16]. Moreover, $\mathrm{CO}$ has anti-inflammatory activity e.g. by inhibiting the inflammatory response of donor Kupffer cells upon transplantation [17], activation of anti-inflammatory mitogen-activated protein kinase signaling [18], and upregulation of the anti-inflammatory cytokine IL-10 [19]. Furthermore, $\mathrm{CO}$ inhibits the acquisition of a pro-adhesive phenotype of vascular endothelial cells [20].

Most studies on the role of $\mathrm{CO}$ in the amelioration of inflammatory responses have been performed by administration of gaseous $\mathrm{CO}$ [21]. However, the applicability of gaseous $\mathrm{CO}$ is limited by its toxic effect on cellular respiration. Therefore, therapeutic use of $\mathrm{CO}$ as a cytoprotective agent clearly requires a pharmaceutical formulation that allows for the selective delivery and/or local release of $\mathrm{CO}$ from a non-toxic pro-drug. In this respect, transitional metal carbonyl-based compounds are of particular interest because of their capability for controlled release of $\mathrm{CO}$ in biological systems [22]. These so-called COreleasing molecules (CORMs) have been therapeutically tested in a variety of experimental inflammatory models with promising anti-inflammatory responses [12,23,24]. Indeed, treatment of septic mice with tricarbonyldichlororuthenium (II) dimer (CORM-2) strongly attenuated liver inflammation, as evidenced by a decrease in serum liver damage markers and a reduced influx of inflammatory cells[25]. In addition, CORM-2 was recently reported to improve outcome of ischemia reperfusion injury to the small bowel [26]. Importantly, CORM treatment is associated with low or minimal formation of carboxyhemoglobin and is therefore considered a safer alternative to $\mathrm{CO}$ gas inhalation [22].

Based on the above, we hypothesized that CORM-2 may ameliorate damage occurring during hepatic I/Ri. Here, we investigated this hypothesis in a rat model of experimentally induced hepatic I/Ri.

\section{Methods}

\section{Rats and experimental procedure}

All animal experiments were performed in accordance with the experimental protocol approved by the Committee for Research and Animal Ethics of Harbin Medical University. For the experiments, healthy male Wistar rats ( $\mathrm{n}=40$; body weight, 230-250 g) were purchased from the Central Animal Facility of the First Clinical Hospital of Harbin Medical University (Harbin, China). Rats were housed in cages under standard animal care conditions and fed with rat chow ad libitum. All surgical procedures were performed under general anesthesia using sodium pentobarbital $(50 \mathrm{mg} / \mathrm{kg}$ i.p.). Normothermic partial hepatic ischemia was induced by performing a midline laparotomy, exposing the liver hilum and subsequent clamping of portal structures to the left and median lobes with a microvascular clip, yielding $~ 70 \%$ hepatic ischemia [27]. The abdomen was covered during the ischemic period. After $60 \mathrm{~min}$ of partial hepatic ischemia, the clip was removed to initiate hepatic reperfusion and the abdominal cavity was closed with a 4-0 silk suture. Temperature was maintained at $37^{\circ} \mathrm{C}$ by a warming pad. Sham-operated rats underwent the same procedure without clamping the pedicle of the liver lobes.

Rats were randomly assigned into four groups with a sample size of 10. Sham group underwent a sham operation and received saline. I/R group underwent the hepatic $\mathrm{I} / \mathrm{R}$ procedure and received saline. CORM-2 group underwent the same procedure and received $8 \mathrm{mg} / \mathrm{kg}$ of CORM-2 (Sigma-Aldrich, St. Louis, MO). iCORM-2 group underwent the same procedure and received iCORM-2 $(8 \mathrm{mg} / \mathrm{kg})$, which does not release CO. All treatments were administered prior to reperfusion. Rats were sacrificed by exsanguination at 6 hours post reperfusion upon which serum and liver samples were col- 
lected according to standard procedures. Rats in all experimental conditions survived the 6 hour reperfusion period.

\section{Serum transaminase determination}

At the end of reperfusion, $5 \mathrm{ml}$ of blood was collected from the caval vein in heparinized tubes. Samples were centrifuges at $800 \mathrm{~g}$ for 10 minutes at room temperature. Plasma was then used to evaluate the extent of hepatic injury by measuring serum levels of ALT and AST using an Automated Chemical Analyzer (7600; Hitachi, Tokyo, Japan). Values were expressed as units per liter (U/L).

\section{Liver histopathology score}

Formalin-fixed liver samples were embedded in paraffin, sectioned into $5-\mu \mathrm{m}$ thickness and stained with hematoxylin-eosin and microscopically inspected to assess inflammation and tissue damage. Histological examination of hepatic tissue damage was performed by two liver pathologists in a blinded fashion. Ten separated microscopic fields were scored on a scale from 0 to 3 as described before [28]. The severity of tissue damage was defined as: grade 0 , minimal or no evidence of injury; grade 1, mild injury consisting of cytoplasmic vacuolation to focal nuclear pyknosis; grade 2, moderate to severe injury with extensive nuclear pyknosis, cytoplasmic hypereosinophilia, and loss of intercellular borders; grade 3 , severe necrosis with disintegration of hepatic cords, hemorrhage, and neutrophil infiltration.

\section{Detection of apoptotic cells by terminal deoxyribonucleotide transferase (TdT)-mediated dUTP nick-end labeling (TUNEL) staining}

TUNEL staining (Roche, Shanghai, China) was applied to paraffin-embedded $5-\mu \mathrm{m}$ liver sections to detect DNA fragmentation as a measure for the number of apoptotic cells. Counterstaining was performed with 4',6-diamidine-2'-phenylindole dihydrochloride (DAPI) dye. Briefly, deparaffinized livers sections were incubated in permeabilization solution $(0.1 \%$ TritonX-100 in $0.1 \%$ sodium citrate) for 2 min on ice, incubated with TUNEL reaction mixture for $60 \mathrm{~min}$ at $37^{\circ} \mathrm{C}$ in the dark and incubated for 4 min with DAPI dye in the dark. The numbers of apoptotic cells and total hepatic cells were counted in ten separated microscopic fields under $400 \times$ magnification. Numbers were then averaged and used to calculate the apoptosis index (AI) according to the previously reported formula: AI $=$ (apoptotic cells/total hepatic cells) $\times 100 \%$ [29].

\section{Myeloperoxidase activity assay}

Myeloperoxidase (MPO), an enzyme found predominantly in azurophilic granules of polymorphonuclear leukocytes, was measured as an index of neutrophil infiltration in the ischemic liver Briefly, liver tissue was homogenized, centrifuged at $12.000 \mathrm{~g}$ for 15 minutes at $4^{\circ} \mathrm{C}$, after which MPO activity was measured using a commercial kit (NJJC Bio Inc., Nanjing, China) according to manufacturer's instructions. Absorbance was measured at $460 \mathrm{~nm}$ with a spectrophotometer. MPO activity was expressed as units per gram protein $(\mathrm{U} / \mathrm{g})$.

\section{Cytokine Analysis}

Serum samples were obtained from rats $6 \mathrm{~h}$ after the I/R procedure, at the time-point of termination, and stored at $-20^{\circ} \mathrm{C}$ until further analysis. Serum samples were analyzed for TNF- $\alpha$ and IL-6 levels using commercially available enzyme-linked immunosorbent assay (ELISA) kits (R\&D Systems, Minneapolis, MN) according to manufacturer's instructions. Cytokine levels were expressed as picogram per $\mathrm{ml}(\mathrm{pg} / \mathrm{ml})$.

\section{Western blot analysis}

Liver whole cell homogenates and nuclear extracts were obtained by lysis of hepatic tissue with the Nuclear Extract Kit (Active Motif, Carlsbad, CA) according to manufacturer instructions. Protein aliquots $(50 \mu \mathrm{g})$ were subsequently separated by SDS-PAGE on $5 \%$ or $10 \%$ acrylamide gels and proteins were electrotransferred to PVDF membrane. Protein levels were visualized by incubation with the following antibodies: bcl-2, caspase-3, ICAM-1, HO-1, NF-kB p65, $\beta$-actin, and Histone H1 (all from Santa Cruz Biotechnology, Inc. Santa Cruz, CA). Specific binding of antibodies was visualised using appropriate horseradish peroxidase-linked secondary antibody followed by detection of chemoluminescence using an enhanced chemoluminescence detection kit (Roche) according to manufacturer's instructions. Blots were stained with anti- $\beta$-actin or Histone $\mathrm{H} 1$ antibody to verify equal protein loading.

\section{Liver nuclear factor-kappa B (NF-KB) activation}

Activation of the transcription factor NF-kB was measured using a commercially available ELISA kit (TransAM $^{\mathrm{m}}$ NF-kB p65 Transcription Factor Assay Kit, Active Motif, Carlsbad, CA) according to manufacturer's instructions. Nuclear protein extract was obtained using Nuclear Extract Kit (Active Motif) according to manufacturer's instruction. Subsequently, $15 \mu \mathrm{g}$ nuclear protein extract was used to assay for NF-kB activation. Values were represented as OD450 $\mathrm{nm}$.

\section{Statistical analysis}

All values are expressed as the mean \pm standard deviation (SD). Data were analyzed by one-way ANOVA followed by the Tukey-Kramer post test. For the semi-quantitative histopathological scores, statistical analysis was performed using the Kruskal-Wallis test followed by the Dunn's post test. $\mathrm{P}<0.05$ was considered to indicate statistical significance. 


\section{Results}

CORM-2 attenuates hepatic ischemia reperfusion injury (I/ Ri)

Endogenous CO, produced in response to stress by HO-1, is an important cellular tool to prevent cytotoxic and proinflammatory effects of ROS. Moreover, in various experimental animal models release of $\mathrm{CO}$ by carbon monoxide releasing molecules (CORM) has shown promising cytoprotective and anti-inflammatory activity [12,23,24]. Therefore, we hypothesized that the local release of $\mathrm{CO}$ using CORM-2 might be used for the prevention or reduction of ROS-mediated hepatic ischemia reperfusion injury (I/Ri). To test this hypothesis we treated rats subjected to I/R with CORM-2 at the time-point of reperfusion. In untreated $I / R$ rats, the plasma levels of serum liver damage markers ALT and AST were significantly increased compared to sham-operated rats (Fig 1A and $1 \mathrm{~B}$, respectively; $\mathrm{p}<0.001$ ), indicative of significant liver hepatocyte injury and alterations in hepatic function by I/ Ri. However, a single systemic administration of CORM2 at the time-point of reperfusion significantly attenuated hepatic I/Ri as evidenced by a significant reduction in ALT and AST levels 6 hours post-reperfusion (Fig. 1A, p $<0.05$ and $\mathrm{B}, \mathrm{p}<0.05)$. Semi-quantitative scoring of histopathological data confirmed that treatment with CORM-2 resulted in a significant reduction in liver injury score of I/Ri rats compared to untreated I/R rats (Fig. 1C,
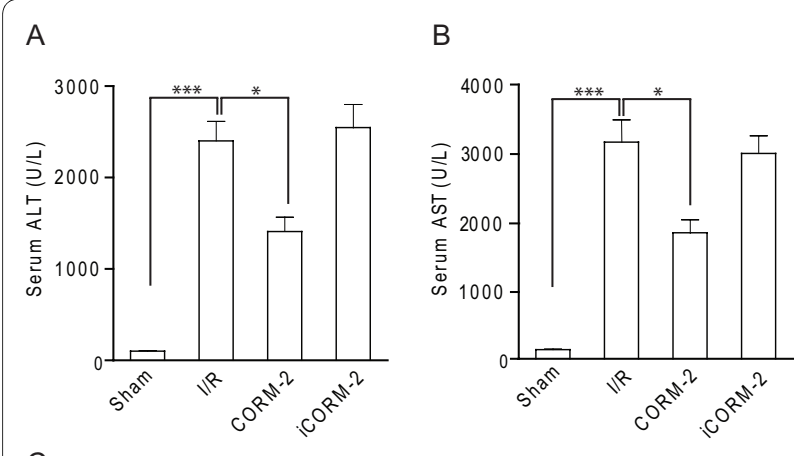

C

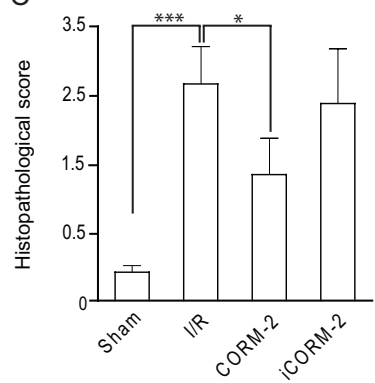

Figure 1 CORM-2 attenuates hepatic ischemia reperfusion injury (I/Ri). Serum levels of $\mathbf{A} A L T$ and $\mathbf{B}$ AST of rats subjected to $60 \mathrm{~min}$. of ischemia followed by $6 \mathrm{~h}$ of reperfusion. $\mathrm{C}$ Liver histology damage scoring of sham-operated rats, I/Ri rats, CORM-2 treated rats and iCORM-2 treated rats. ${ }^{*} \mathrm{P}<0.05$ vs. I/R group. $\mathrm{p}<0.05)$. Of note, although injury score was markedly improved by CORM-2 treatment, it was still elevated compared to sham-operated rats. Importantly, treatment with an inactive form of CORM-2 (iCORM-2), incapable of releasing $\mathrm{CO}$, did not reduce liver $\mathrm{I} / \mathrm{Ri}$, indicating that release of $\mathrm{CO}$ is important for therapeutic activity (Fig. $1 C)$. Taken together, these data clearly demonstrate that $\mathrm{CO}$ released by CORM-2 can ameliorate the negative effects of hepatic I/Ri.

\section{CORM-2 treatment inhibits apoptosis in hepatic I/Ri by up- regulation of $\mathrm{BCl}-2$}

An important consequence of hepatic I/Ri is the loss of hepatocytes due to induction of apoptosis. Earlier studies have shown that inhalation of gaseous $\mathrm{CO}$ can attenuate apoptotic cell death in I/Ri models of the heart [30], lung, kidney [31], and small intestine[32]. Based on these wellestablished cytoprotective effects of CO, we assessed whether CORM-2 treatment reduced the extent of hepatocyte apoptosis in our rat hepatic I/Ri model using TUNEL staining. In non-ischemic livers of sham-operated rats only very few apoptotic cells were observed (Fig. $2 \mathrm{~A}$ ), whereas rats subjected to hepatic I/Ri had a dramatically increased number of apoptotic hepatocytes (Fig. 2B, $\mathrm{p}<0.001)$. Importantly, treatment with CORM-2 markedly reduced the number of apoptotic hepatocytes (Fig. $2 \mathrm{C}, \mathrm{p}<0.05)$. In contrast, treatment of rats with iCORM2 had no significant protective effect, with comparable numbers of TUNEL-stained hepatocytes in the nontreated I/R group and iCORM-2 group (Fig. 2D). Histological data were confirmed by counting apoptotic hepatocytes to obtain an apoptotic index. I/Ri significantly increased the apoptotic index compared to sham-operated rats $(\mathrm{p}<0.001)$. Treatment with CORM-2 significantly reduced the apoptosis index compared to rats subjected to I/Ri (Fig. 2E, p < 0.05). Subsequent Western blot analysis of homogenized liver tissue confirmed that apoptosis was indeed inhibited by CORM-2, as evidenced by a reduction in the level of activation of effector caspase-3 (Fig. 3A and 3B, p < 0.05). Cleaved caspase-3 was strongly present in the I/Ri group and iCORM-2 treated group, whereas caspase-3 cleavage was markedly inhibited in CORM-2 treated rats.

The anti-apoptotic effect of $\mathrm{CO}$ has among others been attributed to up-regulation of anti-apoptotic members and down-regulation of pro-apoptotic members of the Bcl-2 family [31-33]. Indeed, we detected a strong up-regulation of the prototype anti-apoptotic protein Bcl-2 in CORM-2 treated rats when compared to either shamoperated rats (Fig. 3A, p < 0.001) or I/Ri rats (Fig. 3C, p < 0.05). Expression of Bcl-2 in I/Ri-rats was also increased in comparison to sham-operated rats, indicative of the initiation of the tissue homeostatic response. Together, these results indicate that CORM-2 exerts a protective 


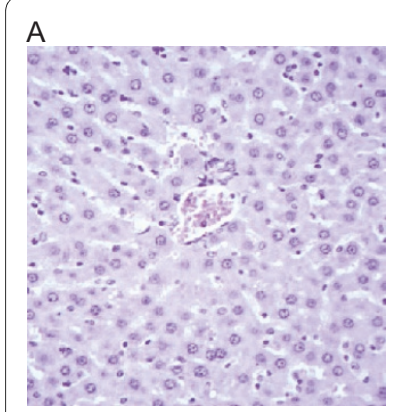

C

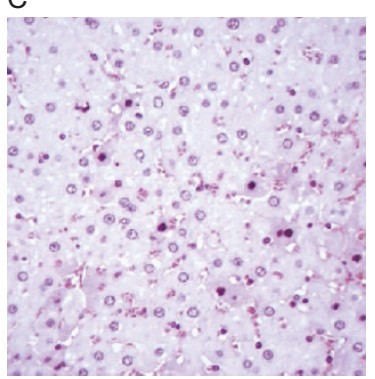

$\mathrm{E}$

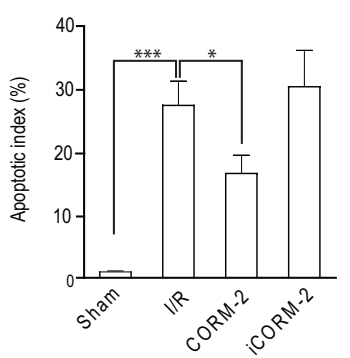

Figure 2 CORM-2 treatment inhibits apoptosis in hepatic I/Ri. Hepatocyte apoptosis was visualised by TUNEL staining in biopsies of $\mathbf{A}$ Sham-operated rats, B I/Ri-treated rats, C CORM-2 treated rats, D iCORM-2 treated rats. E. Graph of the apoptotic index in the various treatment groups. ${ }^{*} P<0.05 \mathrm{vs}$. I/R group.

effect on hepatocytes, at least in part, by up-regulation of $\mathrm{Bcl}-2$ and concomitant inhibition of effector caspase activation.

\section{CORM-2 treatment inhibits production of pro- inflammatory cytokines}

Inflammatory cytokines, such as TNF- $\alpha$, are released by apoptotic and necrotic hepatocytes, vascular endothelial cells and/or Kupffer cells and are known to play key roles in the pathophysiology of hepatic $\mathrm{I} / \mathrm{Ri}[34,35]$. TNF- $\alpha$ is a major inducer of adhesion molecules (i.e. ICAM-1) on vascular endothelial cells and triggers the production of neutrophil attracting CXC chemokines [36,37]. Together, this leads to sinusoidal endothelial cell death and further hepatocyte damage. To determine whether the cytoprotective effect of CORM-2 was associated with a decrease in expression of this important pro-inflammatory mediator, we assessed serum levels of TNF- $\alpha$. In line with litera-
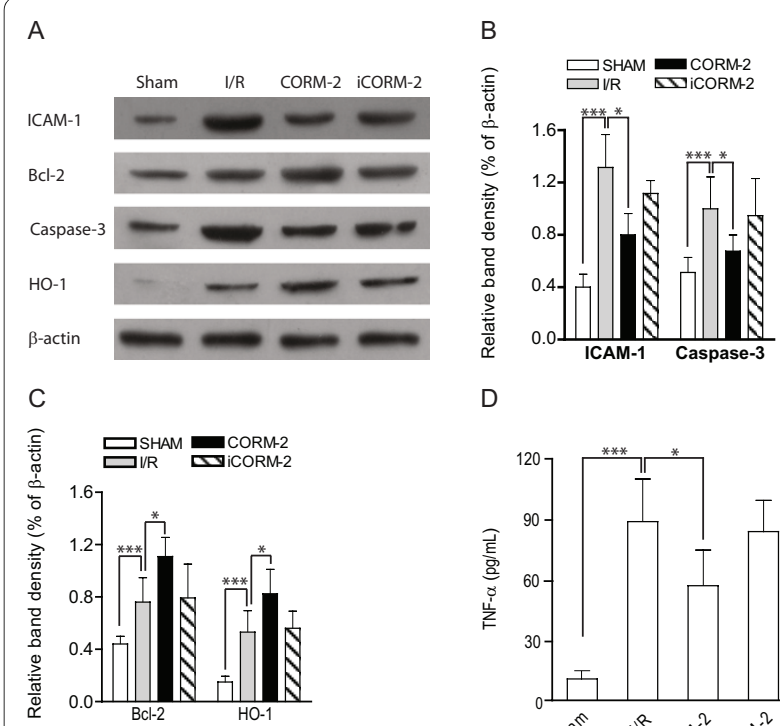

$\mathrm{D}$

E
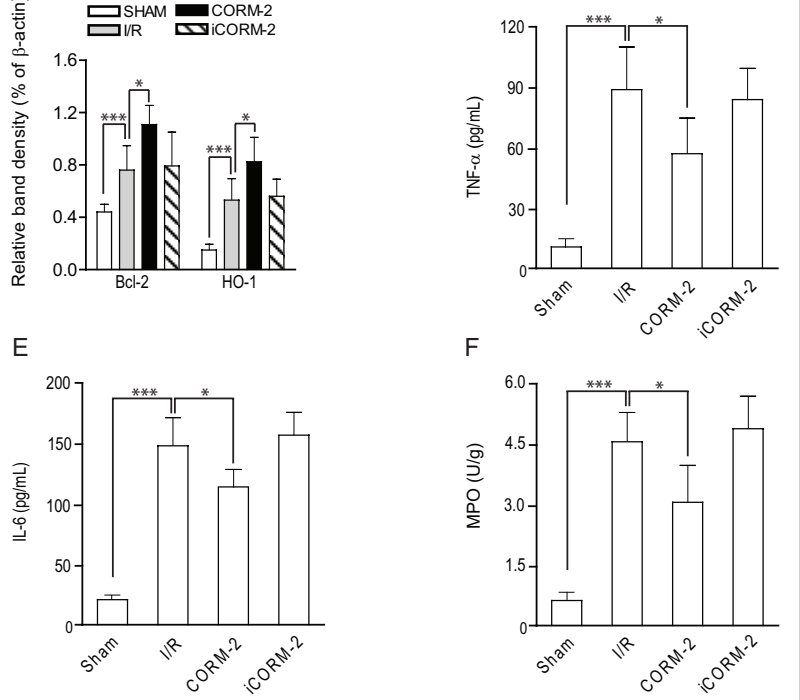

Figure 3 CORM-2 modulates pro- and anti-apoptotic proteins and decreases neutrophil infiltration. A Western blot analysis of the expression of ICAM-1, caspase-3, BCl-2 and HO-1 in sham-operated, I/ Ri operated, CORM-2 treated and iCORM-2 treated rats. $\mathbf{B}$ The relative expression levels of ICAM-1 and caspase- 3 or $\mathbf{C ~ B C l - 2}$ and $\mathrm{HO}-1$ were analysed with densitometry analysis in relation to $\beta$-actin. $\mathbf{D}$ Serum levels of TNF-a and $\mathbf{E}$ IL- 6 of rats subjected to $60 \mathrm{~min}$. of ischemia followed by $6 \mathrm{~h}$ of reperfusion. $\mathbf{F}$ Liver tissue MPO activity for each group was determined. ${ }^{*} P<0.05$ vs. I/R group.

ture, hepatic I/Ri strongly increased serum levels of TNF$\alpha$ compared to base-line levels in sham-operated rats (Fig. $3 \mathrm{D}, \mathrm{p}<0.001)$. This increase in serum levels of TNF- $\alpha$ was significantly inhibited when rats were treated with CORM-2 (Fig. 3D, p < 0.05). In contrast, iCORM-2 did not affect serum levels of TNF- $\alpha$ after I/Ri (Fig. 3D).

Another important cytokine that is produced upon hepatic I/Ri is IL-6, which has long been assumed to play a pivotal role in liver tissue damage and as such is considered to be an important marker for the severity of tissue injury $[38,39]$. In our rat model, hepatic I/Ri induced high serum levels of IL-6 indicative of sever hepatic injury (Fig. $3 E, p<0.001)$. Of note, serum levels of IL- 6 were significantly inhibited by treatment with CORM-2 (Fig. 3E, $\mathrm{p}<$ 0.05). Again, iCORM-2 did not have any effect (Fig. 3E). Thus, the induction of pro-inflammatory cytokines dur- 
ing hepatic $\mathrm{I} / \mathrm{Ri}$ is markedly decreased by treatment with CORM-2.

\section{CORM-2 treatment prevents ICAM-1 expression and decreases neutrophil infiltration}

To further clarify the mechanism of the protective effect of CORM-2 treatment, we assessed whether CORM-2 treatment also had an effect on neutrophil infiltration and activation. An important step in the tissue infiltration of leukocytes is the expression of adhesion molecules, such as ICAM-1, on vascular endothelial cells [40,41]. Indeed, down-regulation of ICAM-1 on vascular endothelial cells can attenuate hepatic I/ $\mathrm{Ri}$ both in vitro and in vivo [42,43]. Several studies have shown that ICAM-1 is essential for leukocyte attachment and infiltration through endothelial cell lining in hepatic sinusoids [44,45]. Our data confirmed that expression of ICAM-1 in the liver was up-regulated as a result of hepatic I/Ri (Fig. 3A and 3B, p < 0.001). Moreover, administration of CORM-2, but not iCORM-2, markedly inhibited the ICAM-1 expression as induced by I/Ri (Fig. $3 \mathrm{~A}$ and $3 \mathrm{~B}, \mathrm{p}<0.05)$.

Next, we assessed whether this reduction in ICAM-1 expression was accompanied by a reduction in neutrophil infiltration. Neutrophil infiltration and activation is an important measure for tissue inflammation and can be quantified by determining tissue myeloperoxidase (MPO) activity [46]. MPO activity in the liver obtained from the I/Ri group was markedly increased compared with livers obtained from sham operated rats (Fig. 3F, p < 0.001). Consistent with the improvement in liver function, the activity of MPO significantly decreased upon CORM-2 administration (Fig. 3F, p < 0.05). In contrast, treatment with iCORM-2 did not affect tissue MPO activity. Thus, the expression of adhesion molecules and the subsequent tissue infiltration of leukocytes, in particular neutrophils, after hepatic I/Ri was effectively reduced by CORM-2 treatment.

\section{CORM-2 blocks pro-inflammatory NF-KB signaling in vivo}

The coordinated induction of hepatocyte apoptosis, the expression of pro-inflammatory cytokines, and the expression of vascular endothelial cell adhesion molecules results in the adhesion and migration of neutrophils and ultimately liver injury. All of these factors are known to be at least partly regulated by the transcription factor NF- $\mathrm{kB}$. The active NF- $\mathrm{BB}$ unit involved in the pro-inflammatory response is the p50/p65 heterodimer, of which the p65 subunit provides the gene regulatory function. In order to evaluate whether the observed beneficial effects of CORM-2 was associated with a down-modulation of NF- $\mathrm{kB}$ activity, we assessed p65 subunit NF- $\mathrm{kB}$ DNA binding activity in hepatic nuclear extracts. I/Ri induced a significant increase in NF-kB p65 DNA binding activity

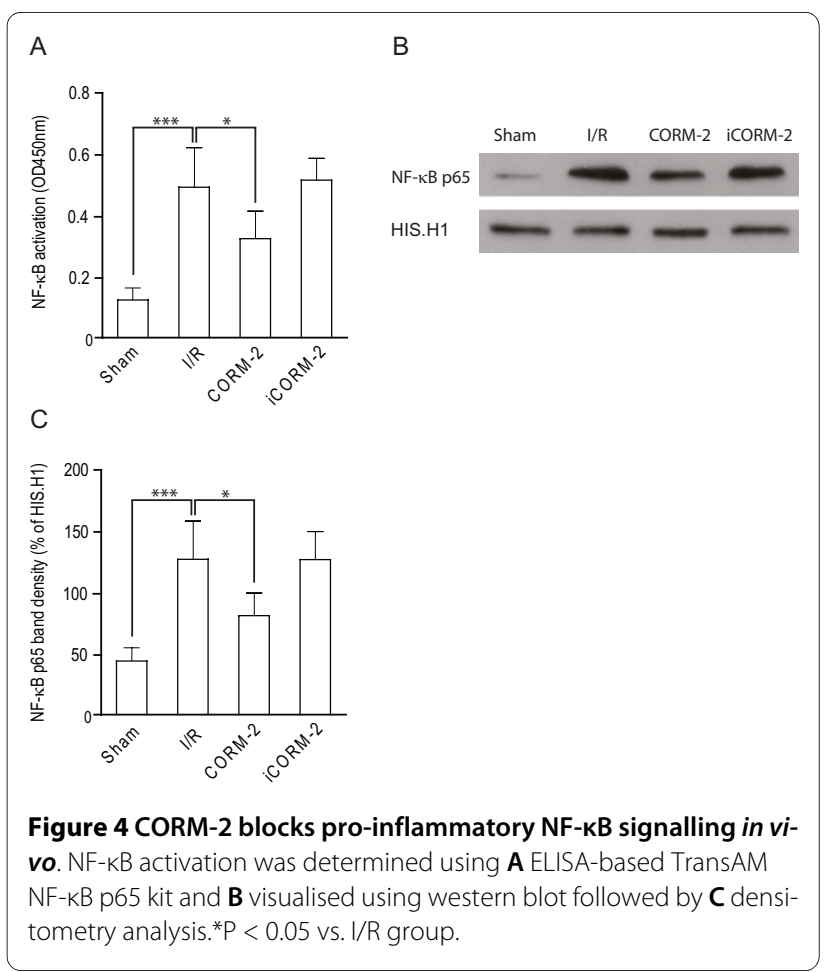

(Fig. 4A, $\mathrm{p}<0.001$ ). This increase in $\mathrm{I} /$ Ri rats was significantly inhibited in hepatic nuclear extracts of I/Ri rats treated with CORM-2 (Fig. 4A, $\mathrm{p}<0.05$ ). In contrast, iCORM-2 had no effect on the activation of NF- $\kappa B$. This effect of CORM-2 treatment on NF- $\mathrm{kB}$ was further confirmed by western blot detection of the presence of the p65 subunit in the nuclear fraction, which revealed a clear increase in protein levels of p65 subunit NF- $\mathrm{kB}$ in untreated $\mathrm{I} / \mathrm{R}$ rats and $\mathrm{iCORM}-2$ treated $\mathrm{I} / \mathrm{R}$ rats (Fig. 4B). However, this translocation was inhibited by treatment with CORM-2 (Fig. 4B). Subsequent quantitation by densitometry revealed that CORM-2 treatment induced a significant reduction in nuclear translocation of the p65 subunit of NF- $\mathrm{kB}$ compared to untreated I/R rats and iCORM-2 treated I/R rats (Fig. 4C, p < 0.05). Taken together, these data indicate that the hepatoprotective effect of CORM-2 was in part due to down-regulation of the inflammatory mediators and inhibition of NF$\kappa B$ activation.

\section{I/Ri-induced HO-1 expression is further augmented by CORM-2 treatment}

The induction of HO-1 is an important cellular stress response that is induced by ischemia reperfusion. Therefore, we next assessed whether HO-1 expression was upregulated by I/Ri and whether CORM-2 treatment had modulatory effects on this stress response. As anticipated, HO-1 expression in the liver obtained from I/Ri group was markedly increased compared to sham-operated animals. Interestingly, CORM-2 but not iCORM-2 
treatment induced a further significant up-regulation of HO-1 (Fig. 3A and 3C, p < 0.05). These data confirm earlier studies, in which activation of the HO system by an HO-1 inducer or by HO-1 gene therapy displayed enhancement of hepatoprotection against warm and cold I/Ri in experimental animals $[47,48]$. Thus, CORM-2 treatment, by inducing local CO-production can help to increase $\mathrm{HO}-1$ expression and thereby further augment the cytoprotective response upon I/Ri.

\section{Discussion}

Endogenous $\mathrm{CO}$ produced by HO-1 is an important cellular protective measure to prevent cytotoxic and proinflammatory effects during reperfusion injury. Here we show that exogenous $\mathrm{CO}$ released by $\mathrm{CO}$-releasing molecule 2 (CORM-2) can be applied to reduce hepatic ischemia reperfusion injury (I/Ri), a common adverse event during liver surgery that is characterized by hepatocellular death and inflammatory cell influx. In our model we demonstrated that CORM-2 treatment reduced the extent of apoptosis and ameliorated the pro-inflammatory stress response as evidenced by a reduction in the expression of pro-inflammatory cytokines, vascular endothelial adhesion molecule and a markedly reduced influx of leukocytes

Importantly, therapeutic application of $\mathrm{CO}$ inhalation is severely hampered by the deleterious effects on the respiratory system due to carboxyhemoglobin formation. For instance, inhalation of $500 \mathrm{ppm}$ gaseous $\mathrm{CO}$ in humans resulted in a peak carboxyhemoglobin level of $7 \%$, whereas in animal studies levels of up to $25 \%$ were detected. In contrast, treatment with CO-releasing molecules such as CORM-2 does not lead to a dramatic increase in carboxyhemoglobin. Indeed, treatment with CORM-2 at doses up to $20 \mu \mathrm{mol} / \mathrm{kg}(10,25 \mathrm{mg} / \mathrm{kg})$ had no negative impact on oxy-haemoglobin saturation [48]. Thus, CORM-2 appears to be a potent inhibitor of negative effects of hepatic I/Ri, while at the same time having no appreciable negative effects on the respiratory system.

Both the cytoprotective and anti-inflammatory activity of $\mathrm{CO}$ appear to result, at least in part, from its ability to modulate the transcription factor NF- $\mathrm{kB}$. For instance, CO-treatment of hepatocytes induces activation of NF$\mathrm{KB}$ in vitro and in vivo [33], which renders these cells more resistant to apoptosis by up-regulating the antiapoptotic protein $\mathrm{Bcl}-\mathrm{xL}$ and down-regulating the proapoptotic protein Bax [49]. Similarly, we found a marked up-regulation of the anti-apoptotic protein Bcl-2 upon CORM-2 treatment. Since the balance between pro- and anti-apoptotic members of the Bcl-2 family is central to the control of the mitochondrial pathway of apoptosis, this increase in Bcl-2 expression is likely to inhibit execution of mitochondrial apoptosis. Of note, pre-treatment of LPS-stimulated human umbilical vein endothelial cells
(HUVEC) with CO showed a reverse effect, namely inhibition of NF- $\mathrm{KB}$ activity [50]. As a result, CO-treated endothelial cells showed a reduced expression of adhesion molecules, which may reduce pro-inflammatory processes such as leukocyte adhesion and tissue infiltration of inflammatory cells. Thus, $\mathrm{CO}$ can have opposite effects on NF- $\mathrm{KB}$ signaling depending on the particular cell type involved. Further detailed investigation, using e.g. laser dissection microscopy may yield insight into the effect of $\mathrm{CO}$ on hepatocytes and hepatic vascular endothelium in vivo. However, from the above it is clear that these diverse effects on NF- $\mathrm{kB}$ cooperate to ameliorate cell damage and minimize inflammation.

In addition to NF-kB, protective effects of $\mathrm{CO}$-released from CORM-2 may be related to the down-regulation of the iNOS/NO pathway in e.g. macrophages. In vitro treatment of LPS-stimulated macrophages with $\mathrm{CO}$ indeed prevented expression of iNOS and blocked the pro-adhesive phenotype $[24,51]$. Furthermore, treatment of $\mathrm{I} / \mathrm{Ri}$ in a rat liver transplantation model using gaseous $\mathrm{CO}$ was partly attributable to down-regulation of iNOS/ NO [16].

As anticipated, the induction of pro-inflammatory cytokines such as TNF- $\alpha$ during hepatic I/Ri is markedly decreased by treatment with CORM-2. Together with the accompanying decrease in expression of adhesion molecules these effects are likely accountable for the reduction in influx of inflammatory cells. The exact mechanism for down-regulation of TNF- $\alpha$ by CORM-2 treatment is still a matter of debate. Various reports have indicated that this effect might be attributable to direct $\mathrm{CO}$-effects on vascular endothelium and circulating leukocytes. Indeed, $\mathrm{CO}$ has potent anti-inflammatory effects on LPS-stimulated HUVEC cells and macrophages [24,25,51]. Another possible contributing factor to the reduction in TNF- $\alpha$ level upon CORM-2 treatment is the rescue of hepatocytes from apoptosis. Apoptosis of hepatocytes is a universal feature of liver inflammation and is associated with the production of various inflammatory cytokines. Thus, the marked reduction in apoptotic hepatocytes upon CORM-2 treatment might contribute to the downplaying of the inflammatory response.

Of note, exogenous application of CORM-2 had an augmenting effect on the expression levels of $\mathrm{HO}-1$, indicating that the exogenous addition of one of the reaction products of HO-1 has a positive feed forward effect on HO-1 expression. Since activation of the $\mathrm{HO}$ system by an $\mathrm{HO}-1$ inducer or by $\mathrm{HO}-1$ gene therapy enhances hepatoprotection against warm and cold I/Ri in experimental animals [46,47], HO-1 upregulation upon treatment with CORM-2 may contribute to the beneficial effects on severity of I/Ri. Indeed, products of the HO-1 enzyme such as bilirubin have well-documented cytoprotective and anti-oxidative activity. Further experiments, 
e.g. using specific HO-1 inhibitors such as zink protoporhyrin or OB-14 [52], may be used in conjunction with CORM-2 treatment to determine the relative contribution of these $\mathrm{HO}-1$ products."

\section{Conclusion}

In conclusion, exogenous $\mathrm{CO}$ as released by CORM-2 treatment has a cytoprotective effect during hepatic I/Ri, most likely mediated by the initial attenuation of apoptosis induction, followed by reduced expression of inflammatory mediators and adhesion molecules, and a concomitant decrease in neutrophil infiltration. Together with recent experimental evidence of beneficial effects of CORMs in kidney and small bowel I/Ri [26,53], these data clearly highlight the potential of CO-releasing molecules such as CORM-2 for the prevention or amelioration of I/Ri damage. Therefore, further pre-clinical investigation into the therapeutic applicability of controlled COrelease by CORM-2 for the prevention of $\mathrm{I} / \mathrm{Ri}$ in hepatic surgery is warranted.

\section{Competing interests}

The authors declare that they have no competing interests.

\section{Authors' contributions}

YW carried out acquisition of data. PC carried out acquisition of data and performed statistical analysis. MB carried out acquisition and interpretation of data. WZ designed the study. EB analyzed and interpreted data and wrote the manuscript. WH: participated in coordination and writing. All authors read and approved the final manuscript.

\section{Acknowledgements}

Financially supported by the Dutch Cancer Society (RUG 2009-4355 to E.B, RUG2005-3358 and 2007-3784 to W.H.), the Alexander von Humboldt Foundation (E.B.) and the Plan of Science and Technology Agency of Heilongjiang Province, China (No: GC08C415).

\section{Author Details}

1Third department of General Surgery, First Clinical Hospital Harbin, Harbin Medical University, Harbin 150001, Heilongjiang, China, ${ }^{2}$ Department of Hepatobiliary Cancer Surgery, Tianjin Medical University Cancer Hospital and City Key Laboratory of Cancer Prevention and Therapy, Tianjin 300060, China and ${ }^{3}$ Department of Surgery, Surgical Research Laboratories, University Medical Center Groningen, University of Groningen, Groningen, the Netherlands

Received: 15 December 2009 Accepted: 5 May 2010 Published: 5 May 2010

\section{References}

1. Lee WY, Lee SM: Ischemic preconditioning protects post-ischemic oxidative damage to mitochondria in rat liver. Shock 2005, 24(4):370-5.

2. Sun K, Liu ZS, Sun Q: Role of mitochondria in cell apoptosis during hepatic ischemia-reperfusion injury and protective effect of ischemic postconditioning. World J Gastroenterol 2004, 10(13):1934-8.

3. Carden DL, Granger DN: Pathophysiology of ischaemia-reperfusion injury. J Pathol 2000, 190(3):255-66.

4. Serracino-Inglott F, Habib NA, Mathie RT: Hepatic ischemia-reperfusion injury. Am J Surg 2001, 181(2):160-6.

5. Selzner N, Rudiger H, Graf R, Clavien PA: Protective strategies against ischemic injury of the liver. Gastroenterology 2003, 125(3):917-36.

6. Okajima K, Harada N, Kushimoto S, Uchiba M: Role of microthrombus formation in the development of ischemia/reperfusion-induced liver injury in rats. Thromb Haemost 2002, 88(3):473-80.
7. Bos E, Leuvenink H, Snijder P, Kloosterhuis N, Hillebrands J, Leemans J, et al: Hydrogen Sulfide-Induced Hypometabolism Prevents Renal Ischemia/Reperfusion Injury. J Am Soc Nephrol 2009, 20(9):1901-5.

8. Kang K, Zhao M, Jiang H, Tan G, Pan S, Sun X: Role of hydrogen sulfide in hepatic ischemia-reperfusion-induced injury in rats. Liver Transp/ 2009, 15(10):1306-14

9. Nicoud IB, Knox CD, Jones CM, Anderson CD, Pierce JM, Belous AE, et al:: 2APB protects against liver ischemia-reperfusion injury by reducing cellular and mitochondrial calcium uptake. Am J Physiol Gastrointest Liver Physio/ 2007, 293(3):G623-G630.

10. Anderson CD, Belous A, Pierce J, Nicoud IB, Knox C, Wakata A, et al: Mitochondrial calcium uptake regulates cold preservation-induced Bax translocation and early reperfusion apoptosis. Am J Transplant 2004, 4(3):352-62.

11. Acquaviva R, Lanteri $R$, Li Destri $G$, Caltabiano R, Vanella L, Lanzafame $S$, et al:: Beneficial effects of rutin and $\mathrm{L}$-arginine coadministration in a rat model of liver ischemia-reperfusion injury. Am J Physiol Gastrointest Liver Physio/ 2009, 296(3):G664-G670.

12. Sun B, Sun Z, Jin Q, Chen X: CO-releasing molecules (CORM-2)-liberated $\mathrm{CO}$ attenuates leukocytes infiltration in the renal tissue of thermally injured mice. Int J Biol Sci 2008, 4(3):176-83.

13. Su H, van Dam GM, Buis Cl, Visser DS, Hesselink JW, Schuurs TA, et al. Spatiotemporal expression of heme oxygenase-1 detected by in vivo bioluminescence after hepatic ischemia in HO-1/Luc mice. Liver Transpl 2006, 12(11):1634-9.

14. Amersi F, Shen XD, Anselmo D, Melinek J, lyer S, Southard DJ, et al:: Ex vivo exposure to carbon monoxide prevents hepatic ischemia/reperfusion injury through p38 MAP kinase pathway. Hepatology 2002, 35(4):815-23.

15. Kaizu T, Ikeda A, Nakao A, Tsung A, Toyokawa H, Ueki S, et al:: Protection of transplant-induced hepatic ischemia/reperfusion injury with carbon monoxide via MEK/ERK1/2 pathway downregulation. Am J Physiol Gastrointest Liver Physiol 2008, 294(1):G236-G244.

16. Kaizu T, Nakao A, Tsung A, Toyokawa H, Sahai R, Geller DA, et al: Carbon monoxide inhalation ameliorates cold ischemia/reperfusion injury after rat liver transplantation. Surgery 2005, 138(2):229-35.

17. Tomiyama K, Ikeda A, Ueki S, Nakao A, Stolz DB, Koike Y, et al:: Inhibition of Kupffer cell-mediated early proinflammatory response with carbon monoxide in transplant-induced hepatic ischemia/reperfusion injury in rats. Hepatology 2008, 48(5):1608-20.

18. Otterbein LE, Bach FH, Alam J, Soares M, Tao Lu H, Wysk M, et al:: Carbon monoxide has anti-inflammatory effects involving the mitogenactivated protein kinase pathway. Nat Med 2000, 6(4):422-8.

19. Lee TS, Chau LY: Heme oxygenase-1 mediates the anti-inflammatory effect of interleukin-10 in mice. Nat Med 2002, 8(3):240-6.

20. Soares MP, Seldon MP, Gregoire IP, Vassilevskaia T, Berberat PO, Yu J, et al:: Heme oxygenase-1 modulates the expression of adhesion molecules associated with endothelial cell activation. J Immunol 2004, 172(6):3553-63.

21. Ott MC, Scott JR, Bihari A, Badhwar A, Otterbein LE, Gray DK, et al:: Inhalation of carbon monoxide prevents liver injury and inflammation following hind limb ischemia/reperfusion. FASEB J 2005, 19(1):106-8.

22. Motterlini R: Carbon monoxide-releasing molecules (CO-RMs): vasodilatory, anti-ischaemic and anti-inflammatory activities. Biochem Soc Trans 2007, 35(Pt 5):1142-6.

23. Sun BW, Chen ZY, Chen X, Liu C: Attenuation of leukocytes sequestration by carbon monoxide-releasing molecules: liberated carbon monoxide in the liver of thermally injured mice. J Burn Care Res 2007, 28(1):173-81.

24. Sawle P, Foresti R, Mann BE, Johnson TR, Green CJ, Motterlini R: Carbon monoxide-releasing molecules (CO-RMs) attenuate the inflammatory response elicited by lipopolysaccharide in RAW2647 murine macrophages. Br J Pharmacol 2005, 145(6):800-10.

25. Cepinskas G, Katada K, Bihari A, Potter RF: Carbon monoxide liberated from carbon monoxide-releasing molecule CORM-2 attenuates inflammation in the liver of septic mice. Am J Physiol Gastrointest Liver Physiol 2008, 294(1):G184-G191.

26. Katada K, Bihari A, Mizuguchi S, Yoshida N, Yoshikawa T, Fraser DD, et al:: Carbon Monoxide Liberated from CO-Releasing Molecule (CORM-2) Attenuates Ischemia/Reperfusion (I/R)-Induced Inflammation in the Small Intestine. Inflammation 2010, 33(2):92-100. 
27. Taniguchi M, Uchinami M, Doi K, Yoshida M, Sasaki H, Tamagawa K, et al:: Edaravone reduces ischemia-reperfusion injury mediators in rat liver. $J$ Surg Res 2007, 137(1):69-74

28. Cavalieri B, Perrelli MG, Aragno M, Mastrocola R, Corvetti G, Durazzo M, et al:: Ischemic preconditioning attenuates the oxidant-dependent mechanisms of reperfusion cell damage and death in rat liver. Liver Transp/ 2002, 8(11):990-9.

29. Qian JM, Zhang H, Wu XF, Li GQ, Chen XP, Wu J: Improvement of recipient survival after small size graft liver transplantation in rats with preischemic manipulation or administering antisense against nuclear factor-kappaB. Transp/ Int 2007, 20(9):784-9.

30. Akamatsu Y, Haga M, Tyagi S, Yamashita K, Graca-Souza AV, Ollinger R, et al: Heme oxygenase-1-derived carbon monoxide protects hearts from transplant associated ischemia reperfusion injury. FASEB J 2004 18(6):771-2

31. Neto JS, Nakao A, Kimizuka K, Romanosky AJ, Stolz DB, Uchiyama T, et al: Protection of transplant-induced renal ischemia-reperfusion injury with carbon monoxide. Am J Physiol Renal Physiol 2004, 287(5):F979-F989.

32. Nakao A, Kimizuka K, Stolz DB, Neto IS, Kaizu T, Choi AM et al: Carbon monoxide inhalation protects rat intestinal grafts from ischemia/ reperfusion injury. Am J Pathol 2003, 163(4):1587-98.

33. Zuckerbraun B, Billiar T, Otterbein S, Kim P, Liu F, Choi A, et al:: Carbon Monoxide Protects against Liver Failure through Nitric Oxide-induced Heme Oxygenase 1. J Exp Med 2003, 198(11):1707-16.

34. Colletti LM, Kunkel SL, Walz A, Burdick MD, Kunkel RG, Wilke CA, et al:: The role of cytokine networks in the local liver injury following hepatic ischemia/reperfusion in the rat. Hepatology 1996, 23(3):506-14.

35. Lentsch AB, Yoshidome $H$, Cheadle WG, Miller FN, Edwards M Chemokine involvement in hepatic ischemia/reperfusion injury in mice: roles for macrophage inflammatory protein- 2 and KC. Hepatology 1998, 27(4):1172-7

36. Vollmar B, Glasz J, Menger MD, Messmer K: Leukocytes contribute to hepatic ischemia/reperfusion injury via intercellular adhesion molecule-1-mediated venular adherence. Surgery 1995, 117(2):195-200.

37. Marubayashi S, Oshiro Y, Maeda T, Fukuma K, Okada K, Hinoi T, et al: Protective effect of monoclonal antibodies to adhesion molecules on rat liver ischemia-reperfusion injury. Surgery 1997, 122(1):45-52.

38. Teoh N, Field J, Farrell G: Interleukin- 6 is a key mediator of the hepatoprotective and pro-proliferative effects of ischaemic preconditioning in mice. J Hepatol 2006, 45(1):20-7.

39. Jin X, Zimmers TA, Perez EA, Pierce RH, Zhang Z, Koniaris LG: Paradoxical effects of short- and long-term interleukin- 6 exposure on liver injury and repair. Hepatology 2006, 43(3):474-84

40. Carlos TM, Harlan JM: Leukocyte-endothelial adhesion molecules. Blood 1994, 84(7):2068-101.

41. Klintman D, Schramm R, Menger MD, Thorlacius H: Leukocyte recruitment in hepatic injury: selectin-mediated leukocyte rolling is a prerequisite for CD18-dependent firm adhesion. J Hepatol 2002, 36(1):53-9.

42. Dold S, Laschke MW, Lavasani S, Menger MD, Thorlacius H: Cholestatic liver damage is mediated by lymphocyte function antigen-1dependent recruitment of leukocytes. Surgery 2008, 144(3):385-93.

43. Rajesh M, Pan H, Mukhopadhyay P, Batkai S, Osei-Hyiaman D, Hasko G, et al.: Cannabinoid-2 receptor agonist $\mathrm{HU}-308$ protects against hepatic ischemia/reperfusion injury by attenuating oxidative stress, inflammatory response, and apoptosis. J Leukoc Biol 2007, 82(6):1382-9.

44. Teoh NC, Ito Y, Field J, Bethea NW, Amr D, McCuskey MK, et al:: Diannexin, a novel annexin $\mathrm{V}$ homodimer, provides prolonged protection against hepatic ischemia-reperfusion injury in mice. Gastroenterology 2007, 133(2):632-46

45. Hafez T, Moussa M, Nesim I, Baligh N, Davidson B, Abdul-Hadi A: The effect of intraportal prostaglandin E1 on adhesion molecule expression, inflammatory modulator function, and histology in canine hepatic ischemia/reperfusion injury. J Surg Res 2007, 138(1):88-99.

46. Bradley PP, Priebat DA, Christensen RD, Rothstein G: Measurement of cutaneous inflammation: estimation of neutrophil content with an enzyme marker. J Invest Dermatol 1982, 78(3):206-9.

47. Coito AJ, Buelow R, Shen XD, Amersi F, Moore C, Volk HD, et al:: Heme oxygenase-1 gene transfer inhibits inducible nitric oxide synthase expression and protects genetically fat Zucker rat livers from ischemiareperfusion injury. Transplantation 2002, 74(1):96-102.
48. Kato H, Amersi F, Buelow R, Melinek J, Coito AJ, Ke B, et al:: Heme oxygenase-1 overexpression protects rat livers from ischemia/ reperfusion injury with extended cold preservation. Am J Transplant 2001, 1(2):121-8.

49. Kim H, Loughran P, Kim P, Billiar T, Zuckerbraun B: Carbon monoxide protects hepatocytes from TNF-[alpha]/Actinomycin D by inhibition of the caspase-8-mediated apoptotic pathway. Biochemical and Biophysical Research Communications 2006, 344(4):1172-8

50. Sun B, Zou X, Chen Y, Zhang P, Shi G: Preconditioning of carbon monoxide releasing molecule-derived $\mathrm{CO}$ attenuates LPS-induced activation of HUVEC. Int J Bio/ Sci 2008, 4(5):270-8.

51. Srisook K, Han SS, Choi HS, Li MH, Ueda H, Kim C, et al:: CO from enhanced $\mathrm{HO}$ activity or from CORM-2 inhibits both $\mathrm{O} 2$ - and NO production and downregulates $\mathrm{HO}-1$ expression in LPS-stimulated macrophages. Biochem Pharmacol 2006, 71(3):307-18.

52. Alaoui-Jamali M, Bismar T, Gupta A, Szarek W, Su J, Song W, et al:: A Novel Experimental Heme Oxygenase-1-Targeted Therapy for HormoneRefractory Prostate Cancer. Cancer Res 2009, 69(20):8017-24.

53. Vera T, Henegar J, Drummond H, Rimoldi J, Stec D: Protective Effect of Carbon Monoxide-Releasing Compounds in Ischemia-Induced Acute Renal Failure. J Am Soc Nephrol 2005, 16(4):950-8.

Pre-publication history

The pre-publication history for this paper can be accessed here: http://www.biomedcentral.com/1471-230X/10/42/prepub

doi: $10.1186 / 1471-230 X-10-42$

Cite this article as: Wei et al., Carbon monoxide-Releasing Molecule-2 (CORM-2) attenuates acute hepatic ischemia reperfusion injury in rats $B M C$ Gastroenterology 2010, 10:42

\section{Submit your next manuscript to BioMed Central and take full advantage of:}

- Convenient online submission

- Thorough peer review

- No space constraints or color figure charges

- Immediate publication on acceptance

- Inclusion in PubMed, CAS, Scopus and Google Scholar

- Research which is freely available for redistribution 